\title{
Outpatient prescribing and prophylactic antibiotic use for recurrent urinary tract infections in British Columbia, Canada
}

\author{
Ariana Saatchi, BASc (Hon); Ji Won Yoo; Fawziah Marra, BSc (Pharm), PharmD
}

Faculty of Pharmaceutical Sciences, University of British Columbia, Vancouver, BC, Canada

Cite as: Saatchi A, Yoo JW, Marra F. Outpatient prescribing and prophylactic antibiotic use for recurrent urinary tract infections in British Columbia, Canada. Can Urol Assoc J 2021;15(12):397-404. http://dx.doi.org/10.5489/cuaj.7162

Published online June 22, 2021

See related commentary on page 405

Appendix available at cuaj.ca

\section{Abstract}

Introduction: Urinary tract infection (UTI) is one of the most common infections, has frequent recurrences, and may debilitate quality of life. UTI is considered recurrent if there are three individual cases of UTI within 12 months. The objective of this study was to set a baseline for recurrent urinary tract infections ( $\mathrm{rUTI}$ ) in women, and rUTI-associated antibiotic prescribing in the presence of antimicrobial stewardship efforts.

Methods: Data for rUTI in women were organized through a provincial prescription database, physician billing system, and a consolidation file to combine antibiotic prescribing, diagnoses, and patient demographics. Rates of rUTI cases and prescriptions were examined, and trends of antibiotics were separated by major anatomical therapeutic chemical classes.

Results: A total of 2234903 rUTI-associated prescriptions were dispensed for 674785 rUTI cases from 2008-2018; 2205703 prescriptions were for treatment and 29310 prescriptions were for prophylaxis of rUTI. The prevalence of rUTI cases declined by $59 \%$, while overall rUTI-associated antibiotic prescribing decreased by $73 \%$. The greatest decrease was seen in quinolones $(87 \%)$, while nitrofurantoin became the most common rUTI antibiotic dispensed, accounting for $42 \%$ of prescriptions overall.

Conclusions: Implementation of numerous antimicrobial stewardship efforts may have contributed to the decrease in antibiotic prescribing, particularly for quinolones. In line with local antibiograms and guidelines, nitrofurantoin is the most used antibiotic for rUTI by far, distinctly preferred over other antibiotics secondary to the lack of $E$. coli resistance.

\section{Introduction}

Urinary tract infection (UTI) is one of the most common bacterial infections, typically caused by E. coli, S. saprophyticus, and other enterobacteriaceae. ${ }^{1}$ Women are commonly affected, with more than half experiencing a UTI in their lifetime. ${ }^{2}$ Furthermore, $20-30 \%$ of women who encounter one episode of UTI are expected to experience a recurrence ( $\mathrm{rUTI}$ ), defined as three episodes of UTI within 12 months., ${ }^{2,3}$ Repeat occurrences contribute significantly to worldwide healthcare burden, especially in cost. In 2000, the U.S. relinquished roughly $\$ 2.47$ billion to treat UTI in women. ${ }^{4}$ In 2019, Canada expended \$264 billion for healthcare, the same system in which UTI was the fifth most common reason for emergency department visits and the eighth most common reason for outpatient clinic visits in 2013.5,6

Effective management of $\mathrm{rUTI}$ is critical, as these infections cause significant discomfort for women and deteriorate quality of life. ${ }^{2,7}$ However, current evidence behind best practices for treating or preventing rUTI has changed with time, with most guidelines suggesting long-term prophylactic antibiotics; yet, optimal duration and antibiotic choices are inconclusive. ${ }^{7,8}$ Blind spots in empirical treatment guidelines can contribute to suboptimal or inconsistent treatment and increase the risk of inappropriate antibiotic use. Antimicrobial resistance (AMR) is a current global health crisis, jeopardizing the long-term efficacy of these essential medications. In Canada, the treatment of drug-resistant infections accounts for $\$ 250$ million dollars annually in direct medical costs. ${ }^{9}$ As UTIs are among both the most common and aggressive forms of resistant infections, the delineation and optimization of their treatment is imperative. ${ }^{10}$ Consequences of unchecked AMR encompass increased financial burden in healthcare, as well as losses in GDP, exponential loss of life, and diminished quality of life. Further, an expert panel determined that resistance effects Canadians disparately, with our most vulnerable groups at highest risk. ${ }^{10}$

Recurrent UTI guidelines and community-based antimicrobial stewardship programs, such as Do Bugs Need Drugs? 
(DBND), have been incorporated into evidence-based practice to help Canadian physicians prescribe appropriately. ${ }^{8,11}$ However, the use of local bacterial resistance patterns is also essential when identifying optimal therapeutic choices. ${ }^{12-15}$

The purpose of this population-based study was to examine the characteristics of adult women in British Columbia (BC), who met the criteria for rUTI, and associated use of antibiotics. Annual trends were analyzed to explore outcomes of interest, such as rates of annual rUTI cases, differences in cases between different age groups and rural/urban areas, rates of UTI-associated antibiotic prescribing, and comparing the proportion of treatment to prophylactic antibiotic use.

\section{Methods}

\section{Study databases}

This study was conducted using three provincial databases containing healthcare-related data on patients in BC..$^{16-18}$ PharmaNet is a centralized network connecting pharmacies for all prescriptions filled in the province. ${ }^{19}$ This index was used to collect antibiotic dispensing information, as classified by the Anatomical Therapeutic Chemical (ATC) system. The ATC system has been developed and annually updated by the World Health Organization (WHO) and is an integral part of Health Canada's drug classification. ${ }^{20,21}$ The Medical Services Plan (MSP) is the public health index for physician billing in BC, documenting information regarding physician visits, including diagnostic codes from the ninth revision of International Classification of Diseases (ICD-9). ${ }^{22}$ Patient demographics were pulled from a consolidation file. ${ }^{17}$ Patient confidentiality was maintained using anonymized study IDs to link these databases in lieu of personal health numbers. Data was anonymized and made available to researchers by Population Data BC. All inferences, opinions, and conclusions drawn in this study are those of the authors, and do not reflect the opinions or policies of the Data Steward(s).

\section{Study cohort}

Data extraction included all records for women who were at least 19 years of age and residents of BC between January 1 , 2008, and December 31, 2018. The definition of a woman with an rUTI required at least three individual MSP diagnoses of UTI within a 12-month period. The date of the first UTI diagnosis was the index date for each UTI event. To rule out ineffective treatment for a single UTI infection, only those physician visits $\geq 30$ days apart were counted to identify an event. ${ }^{3,23-25}$ UTI-related diagnoses included: pyelonephritis (590), cystitis (595), and other disorders of urethra and urinary tract (599). ${ }^{26}$
Antibiotic information was extracted from PharmaNet and linked to relevant UTI diagnoses using an algorithm that matched the date of dispensation to an MSP record, within five days. Multiple prescriptions per patient were permitted in the analyses, as patients in the cohort must have received at least three UTI diagnoses within a 12-month period to be included. These prescriptions were differentiated by duration; prescriptions with a duration of 14 days or fewer were considered treatment, since this is the maximum period suggested for treatment, while a duration of 28 days or more was considered prophylaxis, based on trials conducted for rUTI. ${ }^{2,8}$ Any rUTI-associated prescription longer than 28 days was considered prophylactic to exclude treatment prescriptions of inappropriate duration and to include short-term prophylactic prescriptions. Antibiotic classes analyzed included: penicillins [J01C], other beta-lactam antibacterials [J01D], sulfonamides and trimethoprim [J01E], quinolones [J01M], and other antibacterials [J01X].

A postal code-based algorithm was used to determine neighborhood income quintiles, and rural status was identified by a 0 in the second position of the postal code. These methods are standardized by Statistics Canada. ${ }^{27}$

\section{Study outcomes}

All rates were calculated per 1000 population per year, using age and location-specific denominator estimates for the population through statistics BC. ${ }^{28}$ We first looked at rates of rUTI women per 1000 population across age groups (19-49, 50-64, 65-79, 80+), income quintiles, and rural/urban status, to compare subgroup characteristics and trends.

The overall rate of rUTI-associated antibiotic prescriptions, and antibiotic classes and drugs were compared to identify trends and establish the most and least commonly prescribed antibacterials for rUTI. In addition, the ratio of treatment to prophylaxis prescriptions dispensed was analyzed to assess the relative use of prophylaxis in practice, over time. Annual trends of rUTI cases and antibiotic prescribing were measured with generalized linear regression models (Poisson, $\mathrm{a}=0.05$ ). All analyses were conducted through Excel, SAS 9.4 and $R$ version 4.0.3.

\section{Results}

\section{Overall cohort trends}

As shown in Table 1, 674785 women with rUTI were identified during the study period, with the majority of patients aged 19-49 years and living in urban regions; women were equally distributed within the various income quintiles. However, upon applying population denominators, the highest rates of rUTI per 1000 population was concentrated 


\begin{tabular}{|c|c|}
\hline Cohort characteristics (n) & $\begin{array}{c}\text { Overall } \\
(2008-2018)\end{array}$ \\
\hline Total number of UTI patients & 3307765 \\
\hline Mean UTI diagnoses per patient & 8.24 \\
\hline Number of rUTI patients & 674785 \\
\hline \multicolumn{2}{|l|}{ Number of rUTI patients by age } \\
\hline $19-49$ & 309145 \\
\hline $50-64$ & 149115 \\
\hline $65-79$ & 124054 \\
\hline $80+$ & 92471 \\
\hline \multicolumn{2}{|l|}{ Number of rUTI patients by income quintile ${ }^{1}$} \\
\hline Quintile 1 (lowest) & 138196 \\
\hline Quintile 2 & 135949 \\
\hline Quintile 3 & 133785 \\
\hline Quintile 4 & 134828 \\
\hline Quintile 5 (highest) & 123925 \\
\hline Missing $^{3}$ & 8102 \\
\hline \multicolumn{2}{|c|}{ Number of rUTI patients by rural/urban status ${ }^{2}$} \\
\hline Rural & 96341 \\
\hline Urban & 552518 \\
\hline Missing $^{3}$ & 25926 \\
\hline rUTI-associated antibiotic prescriptions & 2234903 \\
\hline \multicolumn{2}{|c|}{$\begin{array}{l}\text { 'Population Data BC determined neighborhood income quintile (i.e., household size- } \\
\text { adjusted measure of household income) using a postal code based algorithm standardizec } \\
\text { by Statistics Canada. }{ }^{2} \text { Rural status represents local population of } 1000 \text { to } 29999 \text {; urban } \\
\text { status represents local population } \geq 30000 \text {. }{ }^{3} \text { Missing represents absent or not applicable } \\
\text { patient demographic information. rUTl: recurrent urinary tract infection. }\end{array}$} \\
\hline
\end{tabular}

among women above 80 years of age, those living in rural regions, and in the lowest income quintile.
Overall, prevalence of rUTI in women decreased by $59 \%$ between 2008 and 2018 ( $p<0.05$ ) (Fig. 1). A decreasing trend of rUTI was identified when women were stratified by age, with the steepest decline occurring in women aged 19-49 years (65\%) between 2008 and 2018. Although rUTI in the elderly, aged 80 years or older, also declined, it was to a lesser degree (45\%) during the study period. Women in all income quintiles experienced a decrease in rates of rUTI by $59-61 \%$ over the study period, with no significant difference in trends found across quintiles (data not shown). With respect to geographic distributions, the number of women with rUTI decreased by $15 \%$ for urban and $17 \%$ for rural regions over the study period (data not shown).

\section{Treatment and prophylaxis dispensing}

Over the study period, the dispensation of treatment antibiotics ( $n=2205703)$ dominated over prophylactic antibiotics ( $n=29$ 310) (Table 2). Treatment antibiotic dispensing decreased by $73 \%$, from 186 prescriptions per 1000 population in 2008, to 50 prescriptions by 2018 . Prophylactic antibiotic dispensing saw a comparable $70 \%$ reduction during the study period (2000: 2/1000; 2018: 0.7/1000) (Table 2). In a comparison of treatment and prophylaxis antibiotic dispensing, there was a decrease of $9 \%$ over the study period (Table 2 ).

\section{Drug and drug class-specific trends}

Over 2234903 prescriptions for oral antibiotics were dispensed for rUTI during the 11-year study period (Table 1).

Fig. 2 details the rUTI-associated prescriptions dispensed, by major ATC class. Overall, rUTIassociated antibiotic use decreased from 188 prescriptions per 1000 population to only 51 by the end of the study period $(p<0.05)$ (Table 2). Quinolones (J01M), the most frequently dispensed rUTI-associated antibiotic class until 2011, had the greatest decrease $(87 \%)$, from 84 to 11 prescriptions per 1000 women over the study period $(p<0.05)$, and this was related to an $86 \%$ decrease in ciprofloxacin dispensing (Table 3). In contrast, other quinolones, including ofloxacin, levofloxacin, moxifloxacin, and particularly norfloxacin, with four prescriptions dispensed per 1000 women in 2008, were rarely used at the end of the study period (Table 3).

Fig. 1. Rate of recurrent urinary tract infections (rUTIs) in women by age from 2008-2018. 
Saatchi et al

\begin{tabular}{lccccccccccc}
\hline \multicolumn{1}{l}{ Table 2. Rate of rUTI-associated antibiotic use over time by prescription type } \\
\hline & $\mathbf{2 0 0 8}$ & $\mathbf{2 0 0 9}$ & $\mathbf{2 0 1 0}$ & $\mathbf{2 0 1 1}$ & $\mathbf{2 0 1 2}$ & $\mathbf{2 0 1 3}$ & $\mathbf{2 0 1 4}$ & $\mathbf{2 0 1 5}$ & $\mathbf{2 0 1 6}$ & $\mathbf{2 0 1 7}$ & $\mathbf{2 0 1 8}$ \\
\hline rUTI-associated prescriptions & 188.10 & 52.28 & 53.05 & 52.05 & 137.38 & 60.37 & 147.68 & 98.43 & 58.09 & 61.90 & 51.04 \\
& 185.84 & 51.14 & 51.95 & 51.01 & 135.86 & 59.50 & 146.18 & 97.56 & 57.36 & 61.06 & 50.34 \\
Treatment & 2.27 & 1.01 & 1.00 & 0.92 & 1.61 & 0.84 & 1.63 & 1.16 & 0.73 & 0.79 & 0.67 \\
Prophylactic $_{\text {Ratio }^{2}}$ & 82.0 & 50.7 & 52.0 & 55.4 & 84.4 & 70.8 & 89.7 & 83.9 & 78.7 & 77.1 & 74.9 \\
\hline
\end{tabular}

'Rates calculated as prescriptions per 1000 population. ${ }^{2}$ Ratio of treatment:prophylactic use. rUTI: recurrent urinary tract infection.

The decline in the quinolone class was followed by an $84 \%$ reduction in the class of sulfonamides and trimethoprim (J01E), from 33 to five prescriptions per 1000 women over the study period ( $\mathrm{p}<0.05)$, attributed to an $85 \%$ decrease in sulfamethoxazole and trimethoprim (SMX/TMP) dispensing (Table 3).

Penicillin (J01C) dispensing decreased by $69 \%$ over the study period $(p<0.05)$. Although use of most penicillin antibiotics decreased by $78-95 \%$, amoxicillin with an enzyme inhibitor increased by 28\% (Table 3). Cephalosporins (J01D) remained consistent, with only a $7 \%$ decrease over the study period $(p<0.05)$, but within this antibiotic class, cephalexin and cefuroxime use decreased by $66 \%$ and $70 \%$, respectively, while cefixime dispensing increased four-fold from 1.2 to 4.7 prescriptions per 1000 women per year over the study period. In 2008, penicillin and cephalosporins were the two antibiotic classes least likely to be dispensed for rUTI, and this remained true for 2018 as well.

The use of J01X drugs decreased by 54\%, from 56 to 26 prescriptions per 1000 women over the study period $(p<0.05)$, related to a decrease of $61 \%$ in use of nitrofurantoin, but fosfomycin use increased from 0.02 to 1.64 prescriptions per 1000 women (Fig. 2, Table 3). In 2008, ciprofloxacin was the most used antibiotic, with 79.2 prescriptions dispensed per 1000 women; however, its use was overtaken by nitrofurantoin

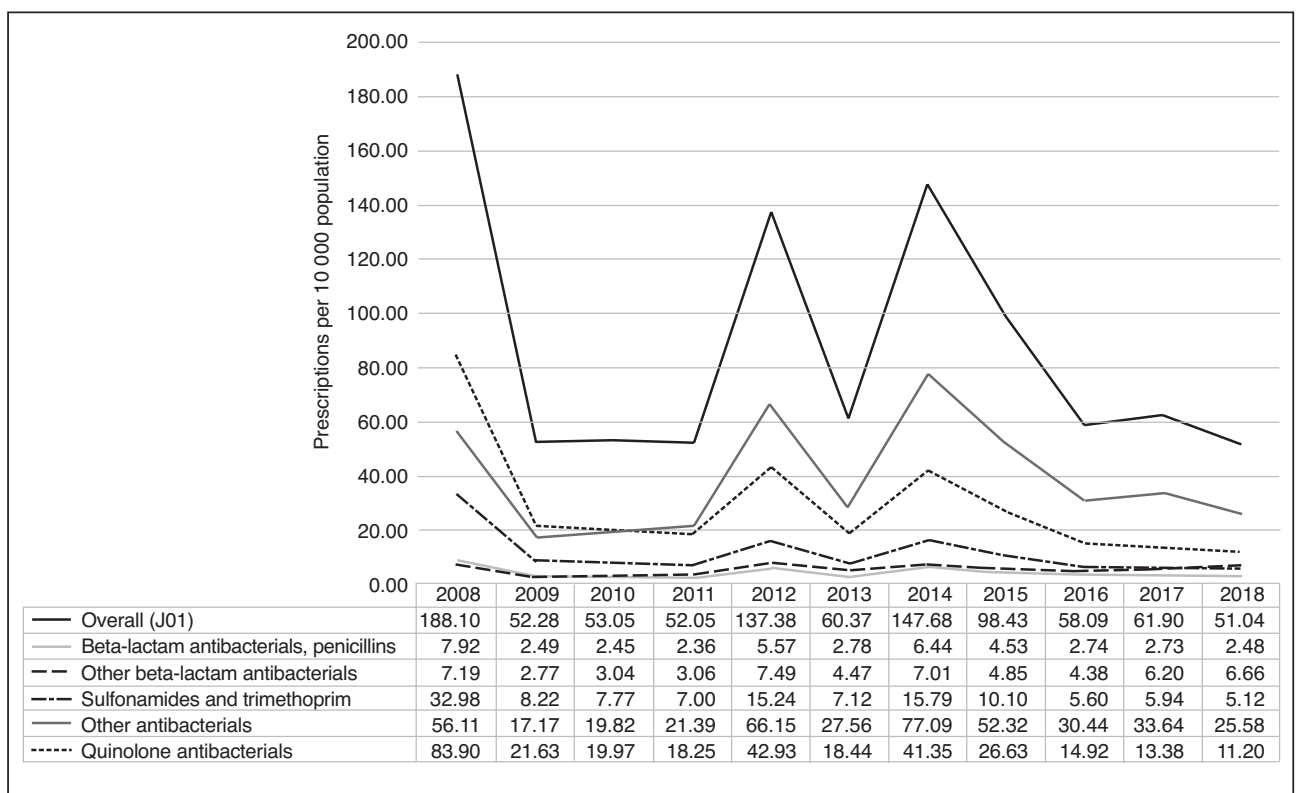

Fig. 2. Rates of antibiotic prescribing by major anatomical therapeutic chemical (ATC) class for women with recurrent urinary tract infections from 2008-2018. from 2010 onward, and in 2018, nitrofurantoin persisted as the most dispensed rUTI-associated antibiotic, with 21.5 prescriptions dispensed per 1000 women in 2018 (Table 3). Still ciprofloxacin remains frequently used, and was the second most dispensed antibiotic for rUTI at the end of the study period, with 11 prescriptions per 1000 women in 2018.

Nitrofurantoin was dispensed the most for both treatment and prophylaxis (Supplementary Figs. 1, 2; available at cuaj.ca). Its dispensation decreased by $61 \%$, from 52.8 to 20.8 treatment prescriptions per 1000 women, and $72 \%$, from 1.25 to 0.35 prophylactic prescriptions per 1000 women over the study period. Prophylactic prescriptions for ciprofloxacin and sulfamethoxazole and trimethoprim (SMX/ TMP) were few in comparison; and dispensation decreased for both antibiotics by approximately $70 \%$, with prophylactic ciprofloxacin dispensing decreasing by $73 \%$, from 0.24 to 0.06 prescriptions per 1000 women, and prophylactic SMX/TMP dispensing decreasing by $72 \%$, from 0.38 to 0.15 prescriptions per 1000 women.

\section{Discussion}

Across 11 years, over 2 million prescriptions for rUTI-associated antibiotics were dispensed to women aged 19 and older. The prevalence of women with rUTI diminished by $59 \%$, and $\mathrm{rUTI}$-associated prescribing declined by $73 \%$ over the study period. Nitrofurantoin accounted for $42 \%$ of prescriptions, whereas ciprofloxacin was dispensed in $31 \%$ of prescriptions. A potential confounder is that a decrease in the diagnosis of rUTI itself would likely decrease the rates of prescribing. However, there was a $34 \%$ decrease in overall prescriptions vs. cases of rUTI, suggesting a decrease in antibiotic use, independent of rates of rUTI.

Variability in decreasing rates of rUTI across age groups can be attributed to advances in the clinical characterizations of various UTIs, which may have pre- 


\begin{tabular}{|c|c|c|c|c|c|c|c|c|c|c|c|}
\hline & 2008 & 2009 & 2010 & 2011 & 2012 & 2013 & 2014 & 2015 & 2016 & 2017 & 2018 \\
\hline \multicolumn{12}{|l|}{ Beta-lactam (J01C) } \\
\hline Phenoxymethylpenicillin & $0.09 \%$ & $0.16 \%$ & $0.14 \%$ & $0.10 \%$ & $0.08 \%$ & $0.07 \%$ & $0.06 \%$ & $0.08 \%$ & $0.09 \%$ & $0.06 \%$ & $0.06 \%$ \\
\hline Amoxicilln & $3.47 \%$ & $3.64 \%$ & $3.53 \%$ & $3.23 \%$ & $2.95 \%$ & $3.25 \%$ & $2.86 \%$ & $2.87 \%$ & $2.89 \%$ & $2.60 \%$ & $2.76 \%$ \\
\hline Ampicillin & $0.20 \%$ & $0.23 \%$ & $0.18 \%$ & $0.15 \%$ & $0.11 \%$ & $0.16 \%$ & $0.10 \%$ & $0.13 \%$ & $0.15 \%$ & $0.09 \%$ & $0.10 \%$ \\
\hline Cloxacillin & $0.04 \%$ & $0.04 \%$ & $0.04 \%$ & $0.05 \%$ & $0.04 \%$ & $0.02 \%$ & $0.02 \%$ & $0.02 \%$ & $0.01 \%$ & $0.03 \%$ & $0.01 \%$ \\
\hline Amoxicillin with enzyme inhibitor & $0.40 \%$ & $0.70 \%$ & $0.69 \%$ & $0.98 \%$ & $0.86 \%$ & $1.09 \%$ & $1.31 \%$ & $1.50 \%$ & $1.57 \%$ & $1.63 \%$ & $1.90 \%$ \\
\hline \multicolumn{12}{|l|}{ Other beta-lactams (J01D) } \\
\hline Cephalexin & $2.67 \%$ & $3.43 \%$ & $3.29 \%$ & $2.92 \%$ & $2.49 \%$ & $2.80 \%$ & $2.46 \%$ & $2.42 \%$ & $2.43 \%$ & $2.73 \%$ & $3.30 \%$ \\
\hline Cefuroxime & $0.48 \%$ & $0.60 \%$ & $0.62 \%$ & $0.71 \%$ & $0.61 \%$ & $0.73 \%$ & $0.73 \%$ & $0.64 \%$ & $0.69 \%$ & $0.62 \%$ & $0.54 \%$ \\
\hline Cefixime & $0.62 \%$ & $1.16 \%$ & $1.79 \%$ & $2.25 \%$ & $2.35 \%$ & $3.87 \%$ & $1.55 \%$ & $1.87 \%$ & $4.42 \%$ & $6.67 \%$ & $9.20 \%$ \\
\hline \multicolumn{12}{|l|}{ Sulfonamides \& trimethoprim (J01E) } \\
\hline Trimethoprim & $0.24 \%$ & $0.32 \%$ & $0.34 \%$ & $0.28 \%$ & $0.19 \%$ & $0.22 \%$ & $0.21 \%$ & $0.16 \%$ & $0.20 \%$ & $0.22 \%$ & $0.21 \%$ \\
\hline Sulfamethoxazole \& trimethoprim & $17.31 \%$ & $15.44 \%$ & $14.32 \%$ & $13.17 \%$ & $10.91 \%$ & $11.58 \%$ & $10.49 \%$ & $10.11 \%$ & $9.46 \%$ & $9.38 \%$ & $9.82 \%$ \\
\hline \multicolumn{12}{|l|}{ Quinolones (J01M) } \\
\hline Ciprofloxacin & $42.17 \%$ & $39.52 \%$ & $36.24 \%$ & $33.87 \%$ & $30.31 \%$ & $29.83 \%$ & $27.51 \%$ & $26.68 \%$ & $25.33 \%$ & $21.28 \%$ & $21.69 \%$ \\
\hline Ofloxacin & $0.05 \%$ & $0.02 \%$ & $0.01 \%$ & $0.01 \%$ & $0.01 \%$ & $0.00 \%$ & $0.01 \%$ & $0.00 \%$ & $0.00 \%$ & $0.00 \%$ & $0.00 \%$ \\
\hline Levofloxacin & $0.19 \%$ & $0.22 \%$ & $0.21 \%$ & $0.18 \%$ & $0.16 \%$ & $0.16 \%$ & $0.09 \%$ & $0.09 \%$ & $0.11 \%$ & $0.12 \%$ & $0.11 \%$ \\
\hline Norfloxacin & $2.12 \%$ & $1.53 \%$ & $0.99 \%$ & $0.84 \%$ & $0.62 \%$ & $0.42 \%$ & $0.29 \%$ & $0.15 \%$ & $0.14 \%$ & $0.10 \%$ & $0.06 \%$ \\
\hline Moxifloxacin & $0.13 \%$ & $0.19 \%$ & $0.25 \%$ & $0.20 \%$ & $0.16 \%$ & $0.16 \%$ & $0.13 \%$ & $0.15 \%$ & $0.12 \%$ & $0.13 \%$ & $0.11 \%$ \\
\hline \multicolumn{12}{|l|}{ Other antibacterial (J01X) } \\
\hline Nitrofurantoin & $29.28 \%$ & $32.09 \%$ & $36.72 \%$ & $40.46 \%$ & $47.45 \%$ & $44.15 \%$ & $49.50 \%$ & $49.37 \%$ & $46.59 \%$ & $47.33 \%$ & $42.16 \%$ \\
\hline Fosfomycin & $0.01 \%$ & $0.08 \%$ & $0.05 \%$ & $0.00 \%$ & $0.04 \%$ & $0.81 \%$ & $2.03 \%$ & $3.13 \%$ & $5.14 \%$ & $6.35 \%$ & $7.21 \%$ \\
\hline Metronidazole & $0.52 \%$ & $0.63 \%$ & $0.57 \%$ & $0.60 \%$ & $0.67 \%$ & $0.69 \%$ & $0.67 \%$ & $0.63 \%$ & $0.66 \%$ & $0.66 \%$ & $0.75 \%$ \\
\hline
\end{tabular}

viously been misdiagnosed as rUTI. For younger women aged $19-49$ years, the $65 \%$ decrease observed may be linked, in part, to an increased awareness of interstitial cystitis/bladder pain syndrome, which can present similarly to rUTI, but does not require antibiotic treatment. ${ }^{29,30}$ In 2016, Canadian guidelines were released to clarify the diagnosis and subsequent treatment of interstitial cystitis/ bladder pain syndrome, which correlate temporally with a decrease in observed cases of rUTI in our study population. ${ }^{31}$ Stewardship efforts to address the issue of inappropriate prescribing for asymptomatic bacteriuria may account for the observed decrease of rUTI in women aged 80 years and above. ${ }^{32-34}$ As this condition does not necessitate antimicrobial use, its proper diagnosis and management is imperative, as suboptimal use of antibiotic treatment and prophylaxis in elderly populations has been associated with increased risk for adverse outcomes. ${ }^{35,36}$

Another promising finding in our study is the low level of prophylactic antibiotics dispensed. The use of prophylactic antibiotics is associated with increased resistance, likely from the constant use of antibiotics over time. ${ }^{37}$ In addition, there is a lack of evidence in the optimal duration and antibiotic choices for prophylaxis, and UTI is known to recur after the termination of prophylaxis. ${ }^{7}$ Infrequent prophylaxis use reflects recommendations to curtail resistance..$^{37}$ However, other factors, such as frequency of infections or recurrence, as well as the sequelae of infections, may also play an integral role in low prophylactic use.

The steep decline in ciprofloxacin use, an $86 \%$ decrease over the study period, is an achievement of significance. Ciprofloxacin, while superior to non-quinolone antibiotics in clinical outcomes when treating uncomplicated UTI in women, is a broad-spectrum antibiotic with severe consequences of resistance and adverse drug events. ${ }^{38,39}$ In addition, an alarming increase in ciprofloxacin-resistant $E$. coli, the most common pathogen in UTI, has been noted across the globe, including British Columbia. ${ }^{40}$ To combat antibiotic resistance and enhance appropriate drug use, the community antimicrobial stewardship program, DBND, had a clinician education program cautioning against fluoroquinolone use but promoting the use of nitrofurantoin, which at the time had only $2.1 \%$ of $E$. coli-resistant isolates. ${ }^{41-43}$

While the dispensation of most antibiotics declined over the study period, amoxicillin-clavulanate, cefixime, and fosfomycin dispensing increased, which may be explained by local beta-lactam susceptibility patterns. ${ }^{44}$ In 2016, 41\% of British Columbia's E. coli isolates were resistant to monotherapy ampicillin, while only $24 \%$ were resistant to amoxicillin/clavulanate and $11 \%$ were resistant to third-generation oral cephalosporin, cefixime. ${ }^{41}$ With superior activity against resistant uropathogens, amoxicillin/clavulanate and cefixime would be preferred. Fosfomycin, on the other hand, has 
been recommended to be used as a first-line UTI antibiotic in the European Society of Clinical Microbiology and Infectious Diseases/Infectious Diseases Society of America (ESMID/IDSA) guidelines of 2011, studied to be as effective as nitrofurantoin in treating UTI. ${ }^{45}$ However, the use of these antibiotics for rUTI are very minimal compared to the use of nitrofurantoin or ciprofloxacin.

BC-specific guidelines released in 2016 for UTI suggest reserving amoxicillin/clavulanate and cefixime for conditions where there is a possibility of increased resistance found in uropathogens. ${ }^{46}$ In addition, ESMID/IDSA guidelines suggest reserving fosfomycin for its efficacy against multidrug-resistant isolates, electing to use nitrofurantoin or SMX/TMP instead. ${ }^{45}$ The findings of our study are in line with guideline recommendations, and indicate appropriate prescribing for $\mathrm{rUTI}$ in $\mathrm{BC}$.

There were no international studies identified that observed the prevalence of women with rUTI and associated prescriptions against which to compare our results. However, a similar Canadian study conducted by Daneman et al examined the use of fluoroquinolones for uncomplicated UTI in women from six Canadian provinces between 2005 and 2015; the provinces examined were BC, Alberta, Saskatchewan, Manitoba, Ontario, and Nova Scotia. ${ }^{38}$ Although they did not evaluate rUTI per se, our findings corroborated Daneman et al, as fluoroquinolones account for approximately one-third of UTI prescriptions, and their use was noted to have diminished over the study period in five of the provinces. ${ }^{38}$

Similarly, a study conducted by Mulder et al in the Netherlands between 1996 and 2014 detailed the effects of guideline implementations in 2005 on female UTI prescribing. ${ }^{47}$ They found that nitrofurantoin use significantly increased, and SMX/TMP use significantly decreased..$^{47}$ However, as the Netherland's guidelines had excluded quinolones from first-line treatment since 1989, quinolone usage was significantly lower than nitrofurantoin usage throughout their study period. ${ }^{47}$

In contrast, the U.S. relies heavily on quinolones. A study comparing UTI-associated prescribing from 2002-2011 found that quinolones are used more frequently than SMX/ TMP or nitrofurantoin for UTI, indicating widespread inappropriate use of antibiotics. ${ }^{48}$ These suggestions are corroborated by Wattengel et al, who found that from 2005-2018 in the U.S., $68 \%$ of outpatients were treated inappropriately for UTI with suboptimal regimens, dose, durations, and drug choice. ${ }^{49}$

Stewardship efforts to combat the crisis of AMR within British Columbia were formalized provincially with the introduction of the DBND campaign in late 2005, ${ }^{11}$ with heavy, early emphases on handwashing and judicious antimicrobial use for respiratory tract infections. The program has since been attributed to curtailing the overuse of antibi- otics related to upper and lower respiratory tract infections, as well as optimizing their prescribing. ${ }^{50}$ Around 2009, the focus of the program turned towards stewardship for UTI prescribing; international and local guidelines, as well as various stewardship materials, were disseminated regarding the judicious use of antibiotics for UTIs. ${ }^{45,51,52}$ Our study period reviews a decade of antimicrobial prescribing in BC and trends reported are reflective of the provincial efforts to optimize UTI prescribing.

However, our analyses do not include rates prior to 2008, and as such, reported trends do not encompass the increasing trends in antibiotic use observed provincially prior to the introduction of the DBND program. ${ }^{44,53}$ The absence of additional years of data results in the reported declines seen in 2008, with more modest changes thereafter. Additional study limitations are relevant to all retrospective studies conducted using administrative data, which is reliant on accurate coding by billing physicians. Our antibiotics data was obtained through linking PharmaNet dispensing and MSP billing within five days of each other, which may exclude self-administered treatment with refills, as these patients would not visit a clinic; therefore, the data may be an underestimation of the true number of prescriptions dispensed.

In addition, as only dispensing data is available, levels of compliance are unknown and unfilled prescriptions are missed. The inclusion of ICD-9 code 599 (other disorders of urethra and urinary tract) may have led to an overestimation of associated antibiotic prescriptions, as this code could be attributed to diagnoses including, but not limited to, UTIs. However, because our definition for rUTI necessitated at least three physician visits within a year, we found it necessary to include this code alongside 590 (pyelonephritis) and 595 (cystitis) in order to ensure maximum case capture - in the absence of well-established definitions for rUTI. Further, as lab data was not available to confirm presence of infection, and comorbidity data were unavailable to delineate higher-risk populations, our use of ICD-9 codes may be subject to misclassification bias.

Future research should use this baseline data to further optimize rUTI-associated prescribing, ensuring continued efficacy and safety of medications used, and directing stewardship efforts. Furthermore, an examination of patient outcomes in tandem to decreasing rates of prescribing is imperative to ensure that reduced antibiotic use is not associated with increased adverse events, like emergency department visits or hospitalizations. Although low levels of prophylactic prescribing are promising, further analyses to delineate associated sequelae are also integral factors to understanding appropriateness in antibiotic use. With increasing rates of antimicrobial resistance and stewardship, this study offers insight as one of the first delineations of rUTI-associated antibiotic use. 


\section{Conclusions}

The achievements of antimicrobial stewardship are reflected through $\mathrm{rUTI}$-associated prescribing in $\mathrm{BC}$ : a decrease in overall prescribing and particularly quinolones, while relatively increasing the overall usage of nitrofurantoin instead, a first-line agent in accordance with guidelines for common types of rUTI. Amoxicillin with enzyme inhibitor, cefixime, and fosfomycin usage have increased, but remain reserved for specific indications, with preference to other agents for most rUTI cases. Prophylactic antibiotic use is rare in comparison to treatment. This suggests improvements in prescribing through the use of local antibiograms and guidelines to ensure optimal efficacy and minimize resistance.

Competing interests: The authors do not report any competing personal or financial interests related to this work.

This paper has been peer-reviewed.

\section{References}

1. Alberta Health Services c1998-2020. Bugs \& Drugs. Females/Healthy Males - First episode. Modified October 16, 2020. Available at: http://www.bugsanddrugs.org/7399B374-C9F6-4044-9E0386B1D11F2874. Accessed Nov. 22, 2020.

2. Foxman B, Barlow R, D'Arcy H, et al. Urinary tract infection: Self-reported incidence and associated costs. Ann Epidemiol 2000;10:509-15. https://doi.org/10.1016/s1047-2797(00)00072-7

3. Glover $M$, Moreira $C G$, Sperandio V, et al. Recurrent urinary tract infections in healthy and non-pregnant women. Urol Sci 2014;25:1-8. https://doi.org/10.1016/i.urols.2013.11.007

4. Griebling TL. Urologic diseases in America project: Trends in resource use for urinary tract infections in women. J Urol 2005;173:1281-7. hitps://doi.org/10.1097/01.ju.0000155596.98780.82

5. Medical Council of Canada. Report of the Incidence and Prevalence of Diseases and Other Health Related Issues in Canada. c2020. Modified April 15, 2013. Available at: https://mcc.ca/wp-content/uploads/ Touchie-Incidence-Prevalence-Report.pdf. Accessed Nov. 22, 2020.

6. Canadian Institute for Health Information. Health spending. c1996-2020 Available at: https://www. cihi.ca/en/health-spending\#: : text=In\%202019\%2C\%20total\%20health\%20expenditure,gross $\% 20$ domestic\%20product\%20(GDP). Accessed Nov. 22, 2020.

7. Albert X, Huertas I, Pereiró II, et al. Antibiotics for preventing recurrent urinary tract infection in non-pregnant women. Cochrane Database Syst Rev 2004;2004:CD001209. hitps://doi.org/10.1002/14651858. cd001209.pub2

8. Anger J, Lee U, Ackerman AL, et al. Recurrent uncomplicated urinary tract infections in women: AUA/ CUA/SUFU guideline. J Urol 2019;202:282-9. https://doi.org/10.1097/ju.0000000000000296

9. Council of Canadian Academies. When antibiotics fail: The expert panel on the potential socio-economic impacts of antimicrobial resistance in Canada. 2019. Available at: http://www.deslibris.ca/ ID/10102747. Accessed Feb. 11, 2021.

10. Public Health Agency of Canada. Canadian Context on Antimicrobial Resistance, CADTH. Published April 17, 2018. Available at: https://www.cadth.ca/sites/default/files/symp-2018/presentations/aprill72018/Concurrent-Session-E6-Antimicrobial-Resistance-Drugs.pdf. Accessed April 14, 2020

11. Alberta Health Services c2020. Do bugs need drugs? Modified Dec.19, 2014. Available at: http://www.dobugsneeddrugs.org/about/. Accessed Nov. 22, 2020.

12. Gunduz $S$, Uluda Altun H. Antibiotic resistance patterns of urinary tract pathogens in Turkish children. Glob Health Res Policy 2018;3:10. https://doi.org/10.1186/s41256-018-0063-1

13. Al-Zahrani J, Al Dossari K, Gabr AH, et al. Antimicrobial resistance patterns of uropathogens isolated from adult women with acute uncomplicated cystitis. BMC Microbiol 2019;19:237. https://doi.org/10.1186/ s12866-019-1612-6

14. Foxman B. The epidemiology of urinary tract infection. Nat Rev Urol 2010;7:653-60. https://doi. org/10.1038/nrurol.2010.190
15. Public Health Agency of Canada. Canadian Antimicrobial Resistance Surveillance System Report. Modified July 9, 2020. Available at: https://www.canada.ca/content/dam/hc-sc/documents/services/drugshealth-products/canadian-antimicrobial-resistance-surveillance-system-2020-report/CARSS-2020-report2020-eng.pdf. Accessed Nov. 22, 2020.

16. British Columbia Ministry of Health [creator]. Consolidation File (MSP Registration \& Premium Billing). V2. Population Data BC [publisher]. Data Extract. MOH, 2011. Available at: http://www.popdata. bc.ca/data. Accessed Nov. 8, 2021.

17. British Columbia Ministry of Health [creator]. Medical Services Plan (MSP) Payment Information File. V2. Population Data BC [publisher]. Data Extract. MOH, 2011. Available at: http://www.popdata.bc.ca/ data. Accessed Nov. 8, 2021.

18. BC Ministry of Health [creator]. PharmaNet. V2. BC Ministry of Health [publisher]. Data Extract. Data Stewardship Committee, 2011. Available at: hittp://www.popdata.bc.ca/data. Accessed Nov. 8, 2021.

19. Province of BC. PharmaCare for B.C. Residents. Available at: https://www2.gov.bc.ca/gov/content/ health/health-drug-coverage/pharmacare-for-bc-residents/pharmanet. Accessed Nov. 22, 2020.

20. World Health Organization. Guidelines for ATC classification and DDD assignment 2020. Modified December 2019 Available at: https://www.whocc.no/filearchive/publications/2020_guidelines_web. pdf. Accessed Nov. 22, 2020

21. Government of Canada. Drug Product Database. Modified May 18, 2015. Available at: https://www. canada.ca/en/health-canada/services/drugs-health-products/drug-products/drug-product-database/ terminology.html. Accessed Nov. 22, 2020.

22. Province of BC. Diagnostic Code Descriptions (ICD-9). Available at: https://www2.gov.bc.ca/gov/ content/health/practitioner-professional-resources/msp/physicians/diagnostic-code-descriptions-icd-9. Accessed Nov. 22, 2020.

23. Copp HL, Yiee JH, Smith A, et al. Urological diseases in America project. Use of urine testing in outpatients treated for urinary tract infection. Pediatrics. 2013;132:437-4. https://doi.org/10.1542/ peds.2012-3135

24. Schneeberger C, Stolk RP, Devries JH, et al. Differences in the pattern of antibiotic prescription profile and recurrence rate for possible urinary tract infections in women with and without diabetes. Diabetes Care 2008;31:1380-5. https://doi.org/10.2337/dc07-2188

25. Alberta Health Services. Bugs \& Drugs. Recurrent cystitis c1998-2020. Modified October 16, 2020. Available at: http://www.bugsanddrugs.org/8F350AEE-EC13-46F9-B42F-674DD6BF2FFE. Accessed Nov. 22, 2020.

26. Province of BC. Diagnostic Code Descriptions (ICD-9). Available at: https://www2.gov.bc.ca/gov/ content/health/practitioner-professional-resources/msp/physicians/diagnostic-code-descriptions-icd-9. Accessed Nov. 22, 2020.

27. Statistics Canada. Postal code conversion file (PCCF), reference guide. Published December 13, 2017. Available at: https://www150.statcan.gc.ca/nl/pub/92-154-g/92-154-g2017001-eng.htm. Accessed Oct. 12, 2020.

28. Province of BC. Population Estimates. Modified July 2021. Available at: https://www2.gov.bc.ca/ gov/content/data/statistics/people-population-community/population/population-estimates. Accessed Nov. 22, 2020.

29. Berry SH, Elliott MN, Suttorp $M$, et al. Prevalence of symptoms of bladder pain syndrome/interstitial cystitis among adult females in the United States. J Urol 2011;186:540-4. https://doi.org/10.1016/i. juro.2011.03.132

30. Berry SH, Bogart LM, Pham C, et al. Development, validation and testing of an epidemiological case definition of interstitial cystitis/painful bladder syndrome. J Urol 2010;183:1848-52. https://doi.org/10.1016/i.juro.2009.12.103

31. Cox A, Golda N, Nadeau G, et al. CUA guideline: Diagnosis and treatment of interstitial cystitis/ bladder pain syndrome. Can Urol Assoc J 2016;10:E136-55. https://doi.org/10.5489/cuaj.3786

32. Nicolle LE, Bradley S, Colgan R, et al. Infectious Diseases Society of America guidelines for the diagnosis and treatment of asymptomatic bacteriuria in adults. Clin Infect Dis Off Publ Infect Dis Soc Am 2005;40:64354. https://doi.org/10.1086/427507

33. Choosing Wisely Canada. Canadian Geriatrics Society list of 5 things physicians and patients should question in geriatrics. Modified November 2020. Available at: https://choosingwiselycanada.org/geriatrics/. Accessed March 4, 2021.

34. Province of British Columbia. UTls in Primary and Long Term Care. Available at: https://www2.gov.bc.ca/ gov/content/health/practitioner-professional-resources/pad-service/utis-in-primary-and-long-term-care. Accessed March 4, 2021.

35. Trautner BW. Asymptomatic bacteriuria: When the treatment is worse than the disease. Nat Rev Urol 2012;9:85-93. https://doi.org/10.1038/nrurol.2011.192

36. Langford BJ, Brown KA, Diong C, et al. The benefits and harms of antibiotic prophylaxis for urinary tract infection in older adults. Clin Infect Dis 2021;73:e782-91. https://doi.org/10.1093/cid/ciab116 
Saatchi et al

37. Fisher H, Oluboyede Y, Chadwick T, et al. Continuous low-dose antibiotic prophylaxis for adults with repeated urinary tract infections (AnTIC): A randomized, open-label trial. Lancet Infect Dis 2018;18:95768. https://doi.org/10.1016/S1473-3099(18)30279-2

38. Daneman N, Chateau D, Dahl $M$, et al. Fluoroquinolone use for uncomplicated urinary tract infections in women: a retrospective cohort study. Clin Microbiol Infect2020;26:613-8. https://doi.org/10.1016/i. cmi.2019.10.016

39. Patrick DM, Hutchinson J. Antibiotic use and population ecology: how you can reduce your "resistance footprint". CMAJ 2009;180:416-21. https://doi.org/10.1503/cmaj.080626

40. Province of BC. Physician's Newsletters. Available at: https://www2.gov.bc.ca/gov/content/health/ practitioner-professional resources/msp/physicians/reference-material/physicians-newsletters. Accessed Nov. 22, 2020

41. BC Centre for Disease Control c2020. Antimicrobial Resistance Summary. Modified 2016. Available at: http://www.bcedc.ca/health-professionals/data-reports/antimicrobial-resistance-utilization/antimicrobialresistance-dashboard. Accessed Nov. 22, 2020.

42. BC Centre for Disease Control c2020. Antimicrobial Resistance Trends in the Province of British Columbic 2014. Modified October 2015. Available at: http://www.bccdc.ca/resource-gallery/Documents/ AMR\%202014\%20Report-3NOV2015_FINAL.pdf. Accessed Nov. 22, 2020.

43. Kranz J, Schmidt S, Lebert C, et al. The 2017 update of the German Clinical Guideline on epidemiology, diagnostics, therapy, prevention, and management of uncomplicated urinary tract infections in adult patients. Part II: Therapy and prevention. Urol Int 2018:100:271-8. https://doi.org/10.1159/000487645

44. BC Centre for Disease Control c2020. Antimicrobial Utilization in the Province of British Columbia 2013. Modified November 2014. Available at: http://www.bccdc.ca/resource-gallery/Documents/Statistics\%20 and\%20Research/Statistics\%20and\%20Reports/Epid/Antibiotics/Reportonantibioticutilization2013_ Nov12_FINAL.pdf. Accessed Nov. 22, 2020.

45. Gupta K, Hooton TM, Naber KG, et al. International clinical practice guidelines for the treatment of acute uncomplicated cystitis and pyelonephritis in women: A 2010 update by the Infectious Diseases Society of America and the European Society for Microbiology and Infectious Diseases. Clin Infect Dis 2011;52:e103 20. https://doi.org/10.1093/cid/ciq257
46. B.C. Provincial Academic Detailing Service. Urinary Tract Infections in Primary Care and Long Term Care. Modified April 2016. Available at: https://www2.gov.bc.ca/assets/gov/health/practitioner-pro/ provincial-academic-detailing-service/pad-uti-empiric-antibiotics.pdf. Accessed Nov. 22, 2020.

47. Mulder $M$, Baan $E$, Verbon $A$, et al. Trends of prescribing antimicrobial drugs for urinary tract infections in primary care in the Netherlands: A population-based cohort study. BMJ Open 2019:9:0027221. https://doi.org/10.1136/bmiopen-2018-027221

48. Kobayashi M, Shapiro DJ, Hersh AL, et al. Outpatient antibiotic prescribing practices for uncomplicated urinary tract infection in women in the United States, 2002-2011. Open Forum Infect Dis 2016 Aug:3:ofw59. https://doi.org/10.1093/ofid/ofw159

49. Wattengel BA, DiTursi S, Schroeck JL, et al. Outpatient antimicrobial stewardship: Targets for urinary tract infections. Am I Infect Control 2020;48:1009-12. https://doi.org/10.1016/i.aiic.2019.12.018

50. McKay RM, Vrbova L, Fuertes E, et al. Evaluation of the do bugs need drugs? Program in British Columbia: Can we curb antibiotic prescribing? Can J Infect Dis Med Microbiol 2011;22:19-24. https://doi.org/10.1155/2011/745090

51. The Medical Services Plan for Medical Practitioners. Province of BC. Physician's Newsletters. Published 2009. Available at: htrps://www2.gov.bc.ca/assets/gov/health/practitioner-pro/medical-services-plan/ july_2009.pdf. Accessed Feb. 20, 2021

52. Patrick DM, Hutchinson J. Antibiotic use and population ecology: How you can reduce your "resistance footprint." CMAJ Can Med Assoc J 2009;180:416-21. https://doi.org/10.1503/cmaj.080626

53. BC Centre for Disease Control. Antimicrobial Resistance Trends in the Province of British Columbia. 2011. Available at: http://www.bccdc.ca/health-professionals/data-reports/antimicrobial-resistance-vilization/ antimicrobial-resistance-dashboard. Accessed Nov. 22, 2020.

Correspondence: Dr. Fawziah Marra, Faculty of Pharmaceutical Sciences, University of British Columbia, Vancouver, BC, Canada; fawziah@mail.ubc.ca 